\title{
Erratum
}

\section{Enhanced antitumor effects of a bicistronic adenovirus vector expressing both herpes simplex virus thymidine kinase and monocyte chemoattractant protein-1 against hepatocellular carcinoma}

\author{
Tomoya Tsuchiyama, Shuichi Kaneko, Yasunari Nakamoto, Yoshio Sakai, Masao Honda, \\ Naofumi Mukaida, and Kenichi Kobayashi
}

Cancer Gene Therapy (2003) 10, 647. doi:10.1038/sj.cgt.7700631

Correction to: Cancer Gene Therapy (2003) 10, 260-269. doi:10.1038/sj.cgt.7700571

In the above paper, an error has been identified in the affiliations of the authors N Mukaida and K Kobayashi. The reference numbers relating to the addresses were transposed and should have appeared as follows:
Naofumi Mukaida, ${ }^{2}$ and Kenichi Kobayashi'

${ }^{1}$ Department of Gastroenterology, Graduate School of Medical Science Kanazawa University, Kanazawa, Japan; and ${ }^{2}$ Division of Molecular Bioregulation, Cancer Research Institute, Kanazawa University, Kanazawa, Japan. 